Applied Remote Sensing

RemoteSensing.SPIEDigitalLibrary.org

\title{
2016 List of Reviewers
}


The Journal of Applied Remote Sensing would like to sincerely thank the following individuals who served as reviewers in 2016. The success of our publication hinges on the voluntary contributions of time and energy put forth by these professionals.

\begin{tabular}{|c|c|}
\hline James Aanstoos & Francesco Bianconi \\
\hline Isaac Abazu & Rajat Bindlish \\
\hline Zulkiflee Abd Latif & Marcelo Biudes \\
\hline Hemn Abdalla & Alfonso Blanco \\
\hline Elfatih Abdel-Rahman & Ian Blanes \\
\hline Saygin Abdikan & Yannick Boucher \\
\hline Maher Aburas & Salah Bourennane \\
\hline Nicola Acito & David G. Bowers \\
\hline Ed Adams & James Boyden \\
\hline Alexander Agathos & Robert Bracken \\
\hline Hemant Kumar Aggarwal & Patrick Brezonik \\
\hline Hossein Aghababaee & Joshua Broadwater \\
\hline Varsha Agrawal & Krishna Buddhiraju \\
\hline Frank Aikman & Merv Budge \\
\hline Thomas Ainsworth & Maria Elena Buemi \\
\hline Ozlem Akar & Shahzad Ahmad Butt \\
\hline Cihan Altuntas & Roberto Camarero \\
\hline ArulMurugan Ambikapathi & Jinde Cao \\
\hline Clark Amerault & Yun-He Cao \\
\hline Ruhul Amin & Facundo Carmona \\
\hline Daoxiang An & Rafael Castanheira de Souza \\
\hline Oleg Angelsky & Lina Castro \\
\hline Albert Ansmann & Jack Cederquist \\
\hline Paul Aplin & Daniele Cerra \\
\hline Erchan Aptoula & Biswajit Chakraborty \\
\hline Aymen Arfaoui & Steven Chan \\
\hline Afshin Asefpour Vakilian & Chein-I Chang \\
\hline Augusto Aubry & Chien-Ping Chang \\
\hline Stefan Auer & Francois Charbonneau \\
\hline Janja Avbelj & Tao Che \\
\hline Alixandre Avila Rodrigues & Daniele Cheloni \\
\hline Suyash Awate & Chen Chen \\
\hline Dogan Aydal & Hao Chen \\
\hline Rodolfo B. Arango & Jianli Chen \\
\hline Alessio Bacci & Lei Chen \\
\hline Charles Bachmann & Quan Chen \\
\hline James Baer & Yang Chen \\
\hline Xueru Bai & Yi-Chin Chen \\
\hline Valerio Baiocchi & Dongcai Cheng \\
\hline John Ball & Eric Dahai Cheng \\
\hline Biplab Banerjee & Jiang Cheng \\
\hline Nisha Bao & Jianghua Cheng \\
\hline Gladimir Baranoski & Tao Cheng \\
\hline Jean-Luc Baray & Tao Cheng \\
\hline William Barnes & Xu Cheng \\
\hline Pier Matteo Barone & Jerzy Chmiel \\
\hline Zaakirah Bassa & Jeganathan Chockalingam \\
\hline Frances Beckett & Pankaj Choudhury \\
\hline Mariana Belgiu & Yunjae Choung \\
\hline Muyinatu Bell & Praveen Chowdary \\
\hline W. Bryan Bell & Tianxing Chu \\
\hline Youcef Bentoutou & Cornell S. L. Chun \\
\hline Fabrizio Berizzi & Igor P. Chunchuzov \\
\hline Segio Bernabe & andrea Ciampalini \\
\hline Jignesh Bhatt & Rok Ciglic \\
\hline Avik Bhattacharya & Pubu Ciren \\
\hline ljaz Bhutta & Ryan Close \\
\hline Xiaoyong Bian & Jeffery Coe \\
\hline
\end{tabular}

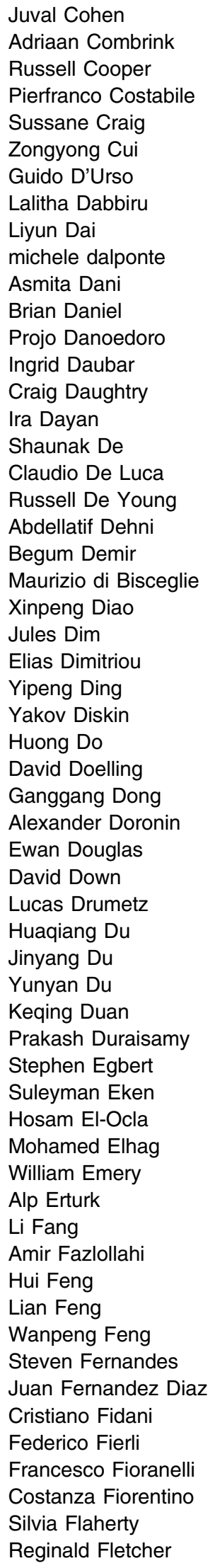


Stefano Fortunati

Samuel Foucher

Bertand Fougnie

Michael Foumelis

Steven Franklin

Frederic Frappart

Andrew French

Katharina Fricke

Cesar Fuentes-Yaco

Frederic Galland

Fabio Gama

Paolo Gamba

Yuquan Gan

Bo-Cai Gao

Feng Gao

Hongyan Gao

Qian Gao

Ruiling Gao

Anne Garnier

Andrea Garzelli

Carol Garzon-Lopez

Rohit Gawande

Song $\mathrm{Ge}$

Sun Genyun

Morteza Ghahremani

Sanjay Ghosh

Surajit Ghosh

Marco Gianinetto

Alex Gilerson

Anatoly Gitelson

Nancy Glenn

Henry Glick

Sertan Gokce

Luis Gomez

Paula Gomez-Perez

Maoguo Gong

Wenyu Gong

Adriana Gonzalez

Sundararaman G. Gopalakrishnan

Richard Gould

Tristan Goulden

Alexander Graham

Raffaele Grasso

John Griffin

Gerhard Groeger

Barry Gross

James H. Gruben

Xiaodong $\mathrm{Gu}$

Haiyan Guan

Huade Guan

Joe Guerci

Arindam Guha

Renzhou Gui

M.Kemal Gullu

Baofeng Guo

Jinyun Guo

Ping Guo

Xian Guo

Yulan Guo

Zhengfu Guo
Dileep Gupta

Mohammed Hachama

Abderrahim Halimi

Youkyung Han

Md. Enamul Haque

Archit Harsh

Mahdi Hasanlou

Quazi Hassan

Ben Haugen

Khaled Hazaymeh

Haiqing $\mathrm{He}$

Lei $\mathrm{He}$

Liming $\mathrm{He}$

Tao He

Yijun $\mathrm{He}$

Muditha Heenkenda

Hans Heidemann

Scott Hensley

Ittai Herrmann

Eric Hetland

Jane Hodgkinson

Dirk Hoffmeister

Saeid Homayouni

Chris Hopkinson

Mehdi Hosseini

Shahram Hosseinzadeh

Biao Hou

Wei Hou

Zhengyang $\mathrm{Hou}$

Allison Howard

Stephan Howden

Jung-Sheng Hsia

Sheng-Jen Hsieh

Lu Hua

Hong Huang

QingNi Huang

Yanbo Huang

David Hulslander

Raymond Hunt

Gianni lannelli

Emmett lentilucci

Francisco Iglesias

Kotaro lizuka

Ikpaya Ikpaya

Tales Imbiriba

Sanaz Imen

Markus Immitzer

Antonio lodice

Muhammad Islam

Muhammad Aminul Islam

Tanvir Islam

Riccardo ludica

Shridhar Jawak

Pradeep Jena

Ryan Jensen

Kun Jia

Xiuping Jia

Zhicheng Jia

Guoyan Jiang

Ruinian Jiang
Xiaoying Jin

Xiuliang Jin

Linhai Jing

Paul E. Johnston

Sushil Joshi

Yue-Dar Jou

Ismail I. Jouny

Kamaruzaman Jusoff

N. Sangeetha Kalaivasan

Akhil Kallepalli

Harry Kambezidis

Xudong Kang

Vassilia Karathanassi

Juha Karvonen

Ingebjorg Kasen

Teerasit Kasetkasem

Jason Kaufman

Mehmet Kavak

Taskin Kavzoglu

Michal Kedzierski

Peter Kennedy

Mahdi Khodadadzadeh

Kourosh Khoshelham

Dennis Killinger

James Kim

Jin-Woo Kim

Yonghyun Kim

Youngwook Kim

Unal Kizil

Kyle Knipper

Takumi Kobayashi

Tomokazu Kobayashi

Dilek Koc-San

Alexander Koltunov

Caitlin Kontgis

Mahen Konwar

Oleg Korablev

Simone Kotthaus

Nikos Koutsias

Samuel Kozaitis

Krzysztof Kulpa

Anil Kumar

Shashi Kumar

Rifat Kurban

Saji Kuriakose

Mehmet Kurum

Tiit Kutser

Ravinder Lall

Salim Lamine

Charis Lanaras

Andreas Langner

Cinzia Lastri

Marco Lavalle

Dong-Cheon Lee

Bob Lempkowski

Javier Leon

Eugene Levin

$\mathrm{He} \mathrm{Li}$

Hongping Li

Huapeng Li 
Hui Li

Jinlong $\mathrm{Li}$

Jun Li

Ning Li

Peijun Li

Rui Li

Shanshan Li

Xi Li

Xiaojie Li

Xiaolu Li

Xinghua Li

Yang Li

Yanyan Li

Yutong $\mathrm{Li}$

Zhaoqin Li

Zhe Li

Zhongming $\mathrm{Li}$

Fengmei Liang

Shouzhen Liang

Xinlian Liang

Tien-Hao Liao

Veraldo Liesenberg

Agnes Lim

Wang-Q Lim

Tang-Huang Lin

Eva Lindberg

Truong-Hong Linh

Bo Liu

Chenguang Liu

Hongying liu

Junzhi Liu

Kui Liu

Kui Liu

Liangyun Liu

Peng Liu

Qiang Liu

Shishi Liu

Sicong Liu

Yinghui Liu

Yuling Liu

Zhaoxia Liu

Zhong Liu

Felipe Lobo

Tim Logan

Bharat Lohani

Laetitia Loncan

Dan Long

Jenny Lovell

Qikai Lu

Rongwen Lu

Xiaomei Lu

Zhong Lu

Maria Lucini

Ben Ludington

Fulin Luo

Xiaoyan Luo

David Lynch

Baoluo Ma

Chao Ma

Ligang Ma
Ghassan Maalouli

Mehdi Maboudi

Sean MacFaden

Bomidi Madhavan

Amir Pasha Mahmoudzadeh

Eva Malinverni

Jordi Mallorqui

Antonino Maltese

Edris Mansouri

Feiyue Mao

Javier Marcello-Ruiz

Maged M. Marghani

Maged Marghany

Armando Marino

Andrea Marinoni

Brian Markham

Vladimir Markov

Prashanth Marpu

Gabriel Martin

Fredrick Mashingia

Antoine Masse

Pedro Mateus

Aneesh Mathew

Vidhya Mathivanan

Stefania Matteoli

Dax Matthews

Igor Meglinski

Alessandro Mei

William Melvin

Tatiana Mendes Sussel

Valeriano Mendez

Xiangchao Meng

Vineetha Menon

Graciela Metternicht

Johannes Meyer

Qiguang Miao

Suresh Middinti

Mario Minacapilli

Kumar Vijay Mishra

Niti Mishra

Harvey Mitchell

Tomoaki Miura

Ali Mohammadzadeh

Helmi Mohd Shafri

Alireza Mohebalhojeh

Rhett Mohler

Alessandro Mondini

Thomas Moranduzzo

Linda Moser

Fred Moshary

Mahdi Motagh

P. Mukhopadhyay

Massimo Musacchio

N. Muthukumaran

Laven Naidoo

Nader Namazi

Ram Narayanan

Jose Nascimento

Ahmed Nashat

Said Nawar
Aditya Nayak

Majid Nazeer

Rogerio Negri

Amy Neuenschwander

Brian Ng

Pham Nghia

Minh Nguyen

Weiping Ni

Giovanni Nico

Doina Nicolae

Konstantinos Nikolakopoulos

Mauricio Noernberg

Imama Noor

Claudia Notarnicola

Artur Nowicki

Hamdan Omar

Thomas Oommen

Natascha Oppelt

Sandip Oza

Caner Ozcan

Asli Ozdarici-Ok

Jayesh Pabari

Jose Padilla-Medina

Nima Pahlevan

Mahesh Pal

Riccardo Palama

Dmitriy Paliy

Prem Pandey

Ioannis Papanikolaou

Matteo Pardini

Sajid Pareeth

Michel Parrot

Ilia Parshakov

N. Patel

Parul Patel

Piyush Pathak

C. Patnaik

Ranjan Patro

William Pearlman

Jifang Pei

Liu Peng

Tao Peng

Antonio Pepe

Monica Pepe

Joao Pereira

Daniel Perez

Maria Perez

Claudio Persello

Raffaele Persico

Dana Petcu

Viet Pham-Bach

Anh Thu Phan

Tuong Phan

Paul Pickell

Stefano Pignatti

Saied Pirasteh

Richard Plant

Jean-Baptiste Poisson

Victor Pomponiu

Octavio Ponce 
Mangalraj Poobalasubramanian

James Porter

Madhukar Potdar

Biswajeet Pradhan

Richa Prajapati

Zuji Qiang

Xianlin Qin

Zhihao Qin

Chunyan Qu

Miguel Quemada

Radhakrishnan Radha

Sudha Radhika

Mahmudur Rahman

Mohammad Rahman

Roozbeh Rajabi

Upendra Rajput

S. Ramachandran

Vinod Ramnath

Abel Ramoelo

Gabriel Ramos-Llorden

T. Rao

Behnood Rasti

Mehul Raval

Mark Ray

Ann Raynal

K. Krishna Reddy

Tamer Refaat

Daniel Reiley

Kevin Repasky

William Reynolds

David Riano

Martina Ricko

Miguel Angel Rico-Ramirez

Haris Riris

Vladimir Risojevic

Christoph Ritter

Gianfranco Rizzo

Dar Roberts

Benjamin Robson

David Roca Bernardez

Pablo Rodriguez-Gonzalvez

Komeil Rokni

Jens Rosebrock

Ronit Rud

Christoph Rudiger

Anand S.

Prasant Sahu

Santo Salinas

Abd El-Azeem Sallam

Bertrand Salquin

Federica Salvetti

Alim Samat

Arockiaraj Samykannu

Nong Sang

Teresa Santos

Lucana Santos Falcon

Malladi Satyanarayana

Michael Schaepman

Paul Scheunders

Christopher Schmidt
Michael Schmitt

Werner Schneider

Mitchell Schull

K. Andrea Scott

Umut Sefercik

Alfonso Senatore

Andres Serna Morales

Apo Sezginer

Reza Shah-Hosseini

Muhammad Shahzad

Shahaboddin Shamshirband

Palanisamy Shanmugam

Hui Shao

Mohammad Sharifikia

J.B. Sharma

Joseph Shaw

Xuhui Shen

Hongbin Shi

Wei Shi

A. S. M. Shihavuddin

Konstantin Shmirko

Greg Showman

Guanyuan Shuai

Mbulisi Sibanda

Till Sieberth

Bastian Siegmann

Markku Similae

Dario Simonetti

Akansha Singh

Keshava Singh

Koushlendra Singh

Ramesh P. Singh

Sudhir Singh

Surendra Singh

Panagiotis Sismanidis

Ramesh Sivanpillai

Duane Smith

G. Louis Smith

M. Sohrabinia

Nieto Solana

Nguyen-Thanh Son

Kaishan Song

Inia Soto Ramos

Jose Sotoca

Hari Shanker Srivastava

Prashant Srivastava

Benoit St-Onge

Michael Starek

Karin Stein

Joshua Stewart

M. Dale Stokes

Richard Stumpf

Hongbo Su

Hongjun Su

Jia Su

Nobuo Sugimoto

Chao Sun

Jing Sun

Jun Qiang Sun

Kang Sun
Liang Sun

Qiang Sun

Qingsong Sun

Sheng Sun

Ting Sun

Wenbo Sun

Robert Sundberg

Olga Sykioti

Szilard Szabo

Subrina Tahsin

Kazunori Takahashi

Jing Tan

Kun Tan

Wenqing Tang

Majid Tangestani

Martha Tapas

Alireza Taravat

Luciano Tarricone

Martin Tauc

Sergio Teggi

Maria Tello Alonso

Pradeep Thapliyal

James Theiler

Prasad Thenkabail

Benjamin Thomas

Valerie Thomas

Liqiao Tian

F. H. C. Tivive

Sonia Tomei

Michelle Tomlinson

Nathan Torbick

Alfonso Torres-Rua

Chiara Torresan

John Trinder

Maxim Truphanov

Eric Truslow

Kuo-Hsin Tseng

Song Tu

Andreas Tzanis

Kabir Uddin

Fawwaz Ulaby

Anwaar Ulhaq

Ruben Usamentiaga

Mustafa Ustuner

Faruk Uysal

Diego Valsesia

Freek van der Meer

Sreenath Rao Vantaram

Carmen Vazquez Garcia

Manuel Veganzones

Vijay Venkataraman

Luisa Verdoliva

Sachin Verghese

Anne Verhoef

Antonio Vettore

Satish Viswanath

Jagath Vithanage

Domenico Vitulano

Gemine Vivone

James Vogelmann 
Michele Volpi

Benoit Vozel

Alaaddin Vural

Edward Waithaka

Philippe Waldteufel

Jeff Walker

Andi Walther

Chao Wang

Cheng-Kai Wang

Cuizhen Wang

Li Wang

Ling Wang

Min Wang

Qingping Wang

Qunming Wang

Robert Wang

Tianxing Wang

Xiaoyang Wang

Yong Wang

Yong Wang

Yongzhe Wang

Zhuosen Wang

Zhuozheng Wang

Zhihui Wei

Pierre Weiss

Xianbin Wen

Ken Whitehead

Pramaditya Wicaksono

Jean-Pierre Williams

Aisheng $\mathrm{Wu}$

Chaoyang Wu

Hongan Wu

Sheng Wu

Wenjin Wu

Xiangqian Wu

Yonghua Wu

Gui-Song Xia

Junshi Xia

Xiang-Gen Xia

Donghui Xie

Huan Xie
Huaping Xu
Jia Xu
Jin Xu
Jingwei Xu
Qizhi Xu
Xiaoyin Xu
Aradhana Yaduvanshi
Hussein Yahia
Fuli Yan
Chenghai Yang
Liang Yang
Qian Yang
Taoli Yang
Xiaohui Yang
Yang Yang
Yi Yang
Yingbao Yang
Zhipeng Ye
Seokwon Yeom
Bingqi Yi
Volkan Yilmaz
Jifu Yin
Ahmad Youssef
Fangfang Yu
Xuelian Yu
Ze Yu
Qiangqiang Yuan
Xiaohui Yuan
Peter Yuen
Yuhendra Yusuf
Klemen Zaksek
Frederic Zamkotsian
Alina Zare
Tanish Zaveri
Tao Zeng
Xingguo Zeng
Wenfeng Zhan
Bing Zhang
Chengye Zhang

Chunhua Zhang

Dongyan Zhang

Guoqing Zhang

Hai Zhang

Jun Zhang

Lei Zhang

Liangpei Zhang

Lifu Zhang

Shaoquan Zhang

Shuqing Zhang

Xiang Zhang

Xiaobo Zhang

Yi Zhang

Ying Zhang

Yingjun Zhang

Yu Zhang

Yunhua Zhang

Zhiming Zhang

Feng Zhao

Hongyu Zhao

Liang Zhao

Tianjie Zhao

Wei Zhao

Zongze Zhao

Guang Zheng

Mingjie Zheng

Zezhong Zheng

Yanfei Zhong

Feng Zhou

Jie Zhou

Daiyin Zhu

Jiahua Zhu

Jie Zhu

Ych Zhu

Zhe Zhu

Richard Zimmerman

Laura Zoffoli

Roghayeh Zoleikani

Qin Zou

Ping Zuo 